\title{
Comparison of Topological Property of Perfect Difference Network and Hypercube
}

\author{
Manisha Singh \\ A.P.S University \\ Computer center, A.P.S.University \\ Rewa (M.P.) 486003
}

\author{
Rekha Tripathi \\ A.P.S University \\ Computer center, A.P.S.University \\ Rewa (M.P.) 486003
}

\begin{abstract}
Perfect difference network are depend on the mathematical notion of perfect difference set.It constitute high performance interconnection networks for parallel and distribution system. Hypercube is loosely coupled parallel processor. The hypercube modeled as a discrete space with discrete time because the processors are in hamming distances. Here we discussed some basic properties of hypercube and PDN
\end{abstract}

\section{INTRODUCTION}

Perfect Difference Network (PDN) that are based on mathematical notation of perfect difference sets, have already been shown to comprise an asymptotically optimal method for connecting a number of nodes into a network with diameter 2.[1]

Hypercube is a loosely coupled parallel processor which already based on the binary n-cube network. It has $n$ dimension and $2 \mathrm{n}$ sides. Here Simple methods are used for calculating the number of nodes "n-2". We have $k$ dimensional hypercube having $\mathrm{N}=2^{\mathrm{k}}$ vertices, so there structure is restricted to having exactly $2^{\mathrm{k}}$ nodes. Hypercube structures size must be power by 2 . Due to the structure sizes there are large gaps in the size of the systems that can be built with the hypercube architecture. [4]

Some topological properties of hypercube make them attractive. Hypercube degree and diameter are equal so its achieve good balance between the communication speed and complexity.

\section{PERFECT DIFFERENCE NETWORK}

Let $\mathrm{x}$ be a $\mathrm{v}$ set of the integer $0.1, \mathrm{v}-1$ module is $\mathrm{V}, \mathrm{D}$ is a set where $D=d_{1} \ldots d_{k} . k$ is a subset of $x$ for every $a \neq 0$ i.e. $\bmod V$, simple difference set for each of the possible difference is formed exactly in $\lambda$ ways

$$
\begin{aligned}
& \text { di, dj, } i \neq j \text { such that } \\
& d i-d j=a(\bmod V)
\end{aligned}
$$

If $\mathrm{A}$ set $\mathrm{D}$ full fill these requirement is called a perfect difference set and the mathematical notion of perfect difference set is create perfect difference network. [5]

PDN is a based on the mathematical notion of PDS by using remainder theorem

$$
\begin{aligned}
& \mathrm{R}=\mathrm{N}-\mathrm{D} \times \mathrm{Q} \\
& \mathrm{M}=\mathrm{R}+\mathrm{D} \times \mathrm{Q} \\
& \mathrm{R}=\mathrm{N} \bmod \mathrm{D}
\end{aligned}
$$

Formula is $\delta \mathbf{i}-\delta \mathrm{j}=$ integer $\bmod \left(\delta^{2}+\delta+1\right)$ here quotient is always one and integer is $1,2,3 \ldots \ldots \ldots \delta^{2}+\delta$

$$
\begin{aligned}
& \text { Integer }=(\delta \mathrm{i}-\delta \mathrm{j})+\left(\delta^{2}+\delta+1\right) \times 1 \\
& \mathrm{i} \text { is the set of }\left\{1,2, \ldots \ldots \ldots \delta^{2}+\delta\right\}
\end{aligned}
$$

\subsection{Perfect Difference Set}

PDS have already been show to comprise an asymptotically optimal method for connecting a number of nodes into a network with diameter 2 .

PDS is a set $\left\{\delta_{0}, \delta_{1}, \delta_{2} \ldots . \delta_{3}\right\}$ of $\delta+1$ integers having the property that their $\delta^{2}+\delta$ differences $\delta \mathrm{i}-\delta \mathrm{j}$

$0<=\mathrm{i}, \mathrm{j}<=\delta$ is congruent module $\delta^{2}+\delta+1$ to the integers $1,2 \ldots \ldots . \delta^{2}+\delta$ in some order is a perfect difference set of order d. [1]

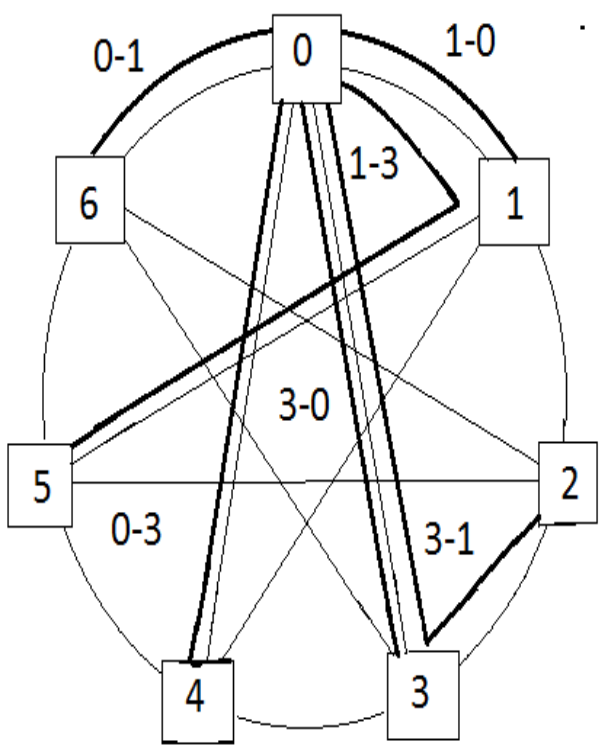

Fig.(1)[7]

(i)

$$
\begin{aligned}
& (1-0)=1 \bmod 7 \\
& N=R+D \times Q \\
& 1=1-0+7 \times 1 \\
& 1=1-0+7
\end{aligned}
$$

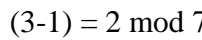

$2=3-1+7 \times 1$

$2=3-1+7$

$2=2+7$

(iii) $\quad(3-0)=3 \bmod 7$ 
$3=3-0+7$

$3=3+7$

(iv)

$(0-3)=4 \bmod 7$

$4=0-3+7 \times 1$

$4=7-3$

$4=4$

(v)

$(1-3)=5 \bmod 7$

$5=(1-3)+7 \times 1$

$5=1-3+7$

$5=7-2$

$5=5$

(vi)

$$
\begin{aligned}
& (0-1)=6 \bmod 7 \\
& 6=0-1+7 \times 1 \\
& 6=0-1+7 \\
& 6=6
\end{aligned}
$$

PDN based on the PDS $\left(0,1,2 \ldots . . \delta_{\delta}\right)$ There are $\mathrm{n}=\delta^{2}+\delta+1$ nodes number 0 to $\mathrm{n}-1$

Node is connected node $\mathrm{i} \pm 1$ and $\mathrm{i} \pm \delta \mathrm{j} \bmod \mathrm{n}$ for $2 \leq \mathrm{j} \delta$ because all index expression in this paper are evaluated module $\mathrm{n}$ throughout.

The basic PDN just define is an undirected Chordal ring of degree $\mathrm{d}=2 \delta$ and diameter $\mathrm{D}=2$. [2]

\section{HYPERCUBE}

A hypercube is a multi dimension mesh it contain each dimension having exactly two nodes. A hypercube has $n$ dimension, where $\mathrm{n}$ can be all positive integer including 0 its consist $2 n$ vertices.

$$
2^{0}=0
$$

$2^{1}=2$

fig.2 (b)

$2^{2}=4$

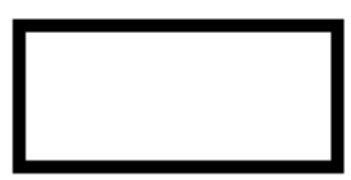

In fig 2 (a) and 2 (b) is a unit hypercube. A hypercube whose dimension is 0 or 1 is called unit hypercube. $\mathrm{n}$ dimensional hypercube is known as $n$ cube.

The number of $\mathrm{m}$ dimensional hypercube on the boundary of an $\mathrm{n}$ cube is
$\mathrm{Em}, \mathrm{n}=2^{\mathrm{n}-\mathrm{m}}(\mathrm{n} \quad \mathrm{m})$, where $(\mathrm{n} \quad \mathrm{m})=$
$\frac{\angle n}{\angle m \angle(n-m)}$ an $\angle \mathrm{n}$ denote the factorial of $\mathrm{n}$ for example

the boundary of a 2 cube so $n=2$ is a vertex in $m$ dimensional boundary. There are $(n, m)$ ways of choosing side, each side is connected $2^{\mathrm{m}}$ times. we need to divide with his number. This number can also be generated by the linear recurrence relation.

$\mathrm{Em} n=2 \mathrm{Em}^{\mathrm{n}-1}+\mathrm{Em}-1, \mathrm{n}-1$, with $\mathrm{E}_{0,0}=1$ and undefined element $=0[6]$

\section{TOPOLOGICAL PROPERTY OF PDN AND HYPERCUBE}

\subsection{Broadcasting}

Distributed memory system processor communicate by message passing .Two neighboring processor exchange data directly, but two remark processor can exchange the data by routing a message through intermediate processor. In a very frequently encountered communication, kernel is the broadcasting of a message, means distribution of same data from one node to all other node. Hear we discuss about several implementation of the broadcast procedure can a hypercube and PDN. [10]

\subsection{Broadcasting in PDN}

Source node sand a message to every other node we consider chordal ring structure of PDN, for $\mathrm{n}=7$, which based on the $\{0,1,3\}$. In fig any two nodes in a PDN are either connected by a link directly or via a path of length 2 , through an inter median node.

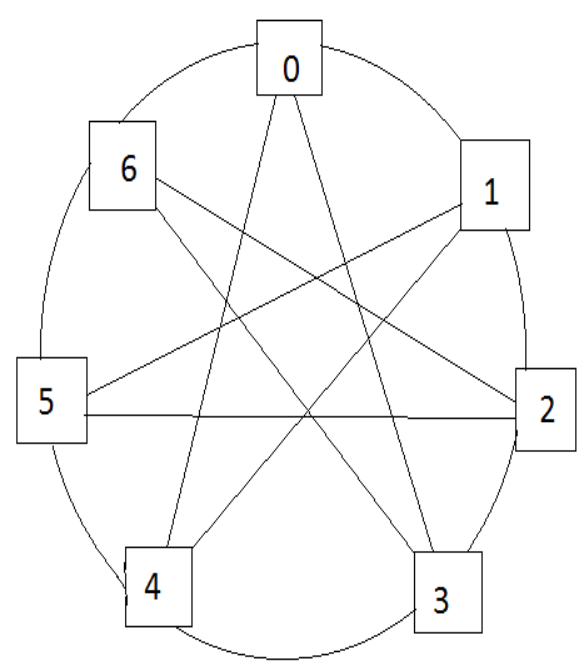

Fig.(3)[1]

One to all maximum message that can by broadcast from any node to all other nodes will be $\delta^{2}+\delta$

One to all broadcasting can be obtained from node 4 to all other node in the table 
Table1. One to all broadcasting

\begin{tabular}{|l|l|l|l|}
\hline $\begin{array}{c}\text { Source } \\
\text { Node }\end{array}$ & \multicolumn{1}{|c|}{$\begin{array}{c}\text { Destination } \\
\text { node }\end{array}$} & \multicolumn{1}{|c|}{ Type of link } & \multicolumn{1}{|c|}{$\begin{array}{c}\text { No. of } \\
\text { hops }\end{array}$} \\
\hline Node 4 & Node 0 & Skip Link & 1 \\
\hline Node 4 & Node 1 & Skip Link & 1 \\
\hline Node 4 & Node 2 & $\begin{array}{l}\text { Ring Link/skip } \\
\text { Link }\end{array}$ & 2 \\
\hline Node 4 & Node 3 & Ring Link & 1 \\
\hline Node 4 & Node 5 & Ring Link & 1 \\
\hline Node 2 & Node 6 & $\begin{array}{l}\text { Ring Link/Skip } \\
\text { Link }\end{array}$ & 2 \\
\hline
\end{tabular}

Summarized the table as follows
(i) $\operatorname{Order}=\delta=2$
(ii) No. of node $=\mathrm{n}=\delta^{2}+\delta+1=7$
(iii) Degree of undirected chordal ring $\mathrm{d}=4$
(iv) Diameter $\mathrm{D}=2$
(v) One to all broadcasting message $\delta^{2}+\delta=6[9]$

\subsection{Broadcasting in hypercube}

Performing the broadcasting in the hypercube is to generate a spanning tree

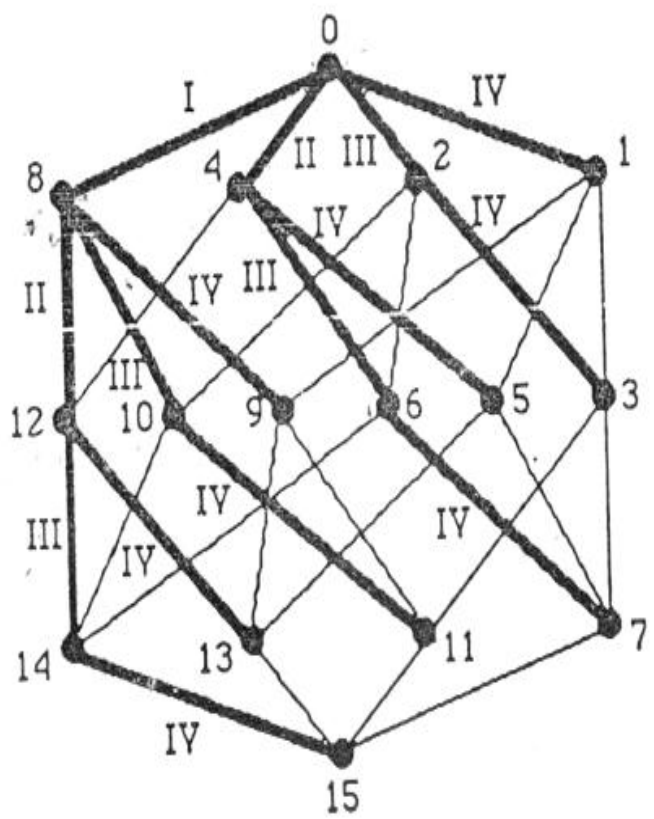

Fig. 4

According to standard binary, reflected gray code, numbered the processors through the length of critical path, of the spanning tree as $\mathrm{m}$.

Let syntax of sending receiving a message between two neighbors is the following

$\{$ Receiving $\}$ receive (hypercube link, receive buffer, length);
\{Sending\} send (hypercube link, send buffer, length);[3]

Here hypercube is dimension along which the message is passed.

In fig. 4 , where $\mathrm{m}=4$, we have follow following step of broadcasting procedure

Step 0- broadcast initiated from processor 0

is to $\mathrm{P}_{8}$

Step 1- Po sends on links dimensions $\mathrm{m}-1=3$, that

Step $2-\mathrm{P}_{0}$ and $\mathrm{P}_{8}$ send on link dimension $\mathrm{m}-2=2$ that is to $\mathrm{P}_{4}$ and $\mathrm{P}_{12}$

Step $3-P_{2}, P_{4}$ and $P_{8}$ send on link dimension $m-1=3$ that is to $\mathrm{P}_{14}, \mathrm{P}_{10}, \mathrm{P}_{6}$ and $\mathrm{P}_{2}$

Step $4-\mathrm{P}_{0}, \mathrm{P}_{2}, \mathrm{P}_{4}, \mathrm{P}_{6}, \mathrm{P}_{8}, \mathrm{P}_{10}, \mathrm{P}_{12}$ and $\mathrm{P}_{14}$ send on link dimension $\mathrm{n}-0=4$ that is to $\mathrm{P}_{1}, \mathrm{P}_{5}, \mathrm{P}_{7}, \mathrm{P}_{9}, \mathrm{P}_{11}, \mathrm{P}_{13}$ and $\mathrm{P}_{15}$

\section{COMPARATIVE STUDYOF PDN AND HYPERCUBE}

The study of topological properties of hypercube and PDN we claim the following result.

(i) PDN provide a large advantage over the hypercube architecture. PDN exist every prime power but hypercube exist only for the power by 2.[8]

(ii) Hypercube have large gaps in size of system then PDN.

(iii) Diameter of hypercube is $\mathrm{n}$ and the diameter of PDN is 2.

(iv) Degree of hypercube is $\mathrm{n}$ and degree of PDN is $2 \delta$.

(v) Hypercube contain 2n node and $\mathrm{n}$ links, but PDN has $\left(\delta^{2}+\delta+1\right)$ node and $\delta\left(\delta^{2}+\delta+1\right)$ link.

(vi) All connection of a hypercube node meets at right angles with respect to each other, where PDN connection makes a chordal ring.

(vii) Hypercube achieve good balance between communication speed and the complexity of the topology network then PDN[3]

\section{CONCLUSION}

We have shown some properties of hypercube and compared them with corresponding properties of PDN. Some topological property of hypercube and PDN is discussed and broadcasting for them was presented. PDN provide a large advantage over the hypercube architecture. PDN exist every prime power but hypercube exist only the power of 2 , so hypercube architecture has a large gaps in the size of system that can be built with the hypercube and its architecture has a delay time approximately equal to $2 \log n$.

\section{REFERENCES}

[1] Katare R.K. Chaudhari N.S. "Study of Topological property of inter connection network and mapping to sparse matrix model.

[2] Parhami B and Rakov MA "Performance algorithmic and robustness attributes of perfect difference network"

[3] Saad, Y. and Schult, M.H. "Topological property of hypercube" IEEE transaction on computers vol 37 no. 7 
[4] Katare, R.K., Chaudhri, N.S. "A comparative study of hypercube and its application to sparse linear system"

[5] Parhami, B. and Rakov M.A. "Application of Perfect difference sets to the designing efficient and Robust inter connection networks.

[6] Ammoj, J. 'Hyper connectivity with in CC Numa architecture' Silicon Graphics. 201 L.N. Shereline Blved. MS 566

[7] Parhami and Rakov M. "Perfect difference networks and related inter connection structures for parallel and distributed system" IEEE trans.
[8] M. Rakov, "Method of inter connection nodes and a hyper star inter connection structure" US Patent No. 573480, Mar. 1998

[9] W.J. Dally and B. Towles "Route Packets not wires: on chip inter connection networks" In Proc. Design automatin cont. (DAC)

[10] M. Rakov, "Muti dimensional hyper star and hyper hub inter connection methods and structure" US Patent application no. 09/410175, Sept. 1999 